\title{
SELFCOMMUTATORS OF MULTICYCLIC HYPONORMAL OPERATORS ARE ALWAYS TRACE CLASS
}

\author{
BY C. A. BERGER ${ }^{1}$ AND B. I. SHAW
}

Communicated by Jacob Feldman, December 9, 1972

1. For $A, B$ operators on the Hilbert space $H,[A, B]=A B-B A$. The selfcommutator of $A$ is $\left[A^{*}, A\right]$. If $E$ is a closed proper subset of the plane, $R(E)$ will be the rational functions analytic on $E$. The operator $A$ is said to be $n$-multicyclic if there are $n$ vectors $g_{1}, \ldots, g_{n} \in H$, called generating vectors, such that $\left\{r(A) g_{i}: r \in R(\operatorname{sp}(A)), 1 \leqq i \leqq n\right\}$ has span dense in $H$. This paper will outline a circle of ideas culminating in the following result.

MAIN THeOREM. If $A$ is an n-multicyclic hyponormal operator, then $\left[A^{*}, A\right]$ is in trace class, and $\operatorname{tr}\left[A^{*}, A\right] \leqq(n / \pi) \omega(\operatorname{sp}(A))$, where $\omega$ is planar Lebesgue measure.

This result is especially interesting because of the scarcity of known conditions insuring that the selfcommutator lie in trace class. The above result is new even when $A$ is subnormal and has a cyclic vector in the usual sense. The best previous result in this direction is due to T. Kato [1], and states that if $\operatorname{Re}(A)$ has finite spectral multiplicity $n$, then $\left[A^{*}, A\right]$ is in trace class. Kato provides a trace estimate which Putnam [4] is able to use to prove the above estimate, where $n$ is an upper bound for the spectral multiplicity of $\operatorname{Re}(A)$.

The Kato-Putnam estimate and the main theorem above are independent. For example, using a result of $\mathrm{J}$. W. Helton and R. Howe, unpublished as yet, which provides a lower bound for the spectral multiplicity of the real part of a hyponormal operator, one can see that the real part of the 1-multicyclic operator given by multiplication by $z$ on $R^{2}$ of a Swiss cheese has infinite spectral multiplicity almost everywhere.

Throughout the following, a space and the orthogonal projection onto that space will be denoted by the same symbol. All spaces are Hilbert spaces.

2. The following lemma is central.

Structure Lemma. Let $T$ and $A$ be hyponormal operators on $H$ and $K$

AMS (MOS) subject classifications (1970). Primary 47B20; Secondary 47B10, 47B47.

Key words and phrases. Hilbert space, trace class, hyponormal operator, selfcommutator, Hankel operator, analytic toeplitz operator.

${ }^{1}$ Research partially supported by grants from the National Science Foundation, NSF GP $32462 X$. 
respectively, and let $W: H \rightarrow K$ be a trace class operator with dense range, such that $W T=A W$. Then $\operatorname{tr}\left[A^{*}, A\right] \leqq \operatorname{tr}\left[T^{*}, T\right]$.

Proof. It may be assumed that $\operatorname{tr}\left[T^{*}, T\right]<\infty$. Let $N$ be the null space of $W$. Since $N$ is an invariant space for $T, T N$ is also hyponormal. It will be shown that $\operatorname{tr}\left[A^{*}, A\right]+\operatorname{tr}\left[N T^{*}, T N\right] \leqq \operatorname{tr}\left[T^{*}, T\right]$.

Let $\left\{\varphi_{n}\right\}_{n}$ be a complete orthonormal system of eigenvectors for $W^{*} W$, with $W^{*} W \varphi_{n}=\lambda_{n}^{2} \varphi_{n}, \lambda_{n} \geqq 0$. Then the vectors $\left\{\psi_{n}: \lambda_{n}>0\right\}$ given by $W \varphi_{n}=\lambda_{n} \psi_{n}$ are a complete orthonormal basis for $K$. Let $L_{t}=H \oplus K$ have the norm $\|h \oplus k\|_{t}^{2}=t^{2}\|h\|^{2}+\|k\|^{2}$, for $t>0$, and let $J$ be the closed subspace spanned by the vectors $\{h \oplus W h: h \in H\}$.

$$
\left\{\left(t^{2}+\lambda_{n}^{2}\right)^{-1 / 2}\left(\varphi_{n} \oplus \lambda_{n} \psi_{n}\right)\right\}_{n}
$$

is a complete orthonormal basis for $J$. Note that $J$ is an invariant space for $T \oplus A$, so $(T \oplus A) J$ is hyponormal. $(T \oplus A) H=(T \oplus 0)$, which, when restricted to $H \oplus 0$, is unitarily equivalent to $T$, so if it can be shown that $H-J$ is in trace class, $\left[J(T \oplus A)^{*},(T \oplus A) J\right]$ will lie in trace class, and

$$
\operatorname{tr}\left[J(T \oplus A)^{*},(T \oplus A) J\right]=\operatorname{tr}\left[H(T \oplus A)^{*},(T \oplus A) H\right]=\operatorname{tr}\left[T^{*}, T\right] .
$$

But the space spanned by the vectors $\left\{\varphi_{n}, \psi_{n}\right\}$ reduces $H-J$, and on this space $H-J$ has trace norm $2 \lambda_{n}\left(t^{2}+\lambda_{n}^{2}\right)^{-1 / 2}$. Thus, $H-J$ has trace norm $\sum_{n} 2 \lambda_{n}\left(t^{2}+\lambda_{n}^{2}\right)^{-1 / 2} \leqq 2 t^{-1} \sum_{n} \lambda_{n}$. Now consider

$$
\begin{aligned}
\operatorname{tr}\left[J(T \oplus A)^{*},(T \oplus A) J\right] \\
=\sum_{\lambda_{n}>0}\left\{\left\|(T \oplus A)\left(t^{2}+\lambda_{n}^{2}\right)^{-1 / 2}\left(\varphi_{n} \oplus \lambda_{n} \psi_{n}\right)\right\|_{t}^{2}\right. \\
\left.\quad-\left\|J\left(T^{*} \oplus A^{*}\right)\left(t^{2}+\lambda_{n}^{2}\right)^{-1 / 2}\left(\varphi_{n} \oplus \lambda_{n} \psi_{n}\right)\right\|_{t}^{2}\right\} \\
\quad+\sum_{\lambda_{n}=0}\left\{\left\|(T \oplus A)\left(t^{-1} \varphi_{n} \oplus 0\right)\right\|_{t}^{2}-J\left(T^{*} \oplus A^{*}\right)\left(t^{-1} \varphi_{n} \oplus 0\right) \|_{t}^{2}\right\} .
\end{aligned}
$$

The diligent reader will discover that the summand in the first sum approaches $\left\|A \psi_{n}\right\|^{2}-\left\|A^{*} \psi_{n}\right\|^{2}$ as $t \rightarrow 0$. (To show that $\|J(0 \oplus u)\|_{t}^{2} \rightarrow$ $\|u\|^{2}$, he will evaluate the norm of the projection using the orthonormal basis for $J$, and apply the Lebesgue monotone convergence theorem to the resulting sum.) A similar technique, applied to the summands of the second sum, and now invoking Lebesgue dominated convergence, shows that they approach

$$
\left\|T \varphi_{n}\right\|^{2}-\sum_{\lambda_{m}=0}\left\{\left|\left\langle T^{*} \varphi_{n}, \varphi_{m}\right\rangle\right|^{2}\right\}=\left\{\left\|T N \varphi_{n}\right\|^{2}-\left\|N T^{*} \varphi_{n}\right\|^{2}\right\} .
$$

Thus, by Fatou's theorem, $\operatorname{tr}\left[A^{*}, A\right]+\operatorname{tr}\left[N T^{*}, T N\right] \leqq \operatorname{tr}\left[T^{*}, T\right]$. 
In light of the Structure Lemma, it is obviously desirable to produce a supple family of hyponormal operators $T$ with trace class selfcommutators.

DEFINITION. For $\mu$ a finite measure with compact support $E$ contained in the compact set $F, R^{2}(F, \mu)$ will be the closure of $R(F)$ in $L^{2}(\mu)$. $R^{2}(E, \mu)$ will be written $R^{2}(\mu)$. If $F$ does not divide the plane, $R^{2}(F, \mu)=$ $H^{2}(\mu) . T_{f}$ on $R^{2}(F, \mu)$ will be the operator $P L_{f} P$, where $P$ is the orthogonal projection on $L^{2}(\mu)$ with range $R^{2}(F, \mu)$.

Computational Lemma. Let $D=\{z:|z|<1\}$, and let $H=$ $H^{2}\left(\chi_{D} \omega\right)$. For $f \in H^{\infty}\left(\chi_{D} \omega\right)$, let $T_{f}=L_{f}$ on $H$, where $L_{f}$ is the Laurent operator. If $f=\sum_{n=0}^{\infty} a_{n} z^{n}$, then

$$
\begin{aligned}
\operatorname{tr}\left[T_{f}^{*}, T_{f}\right] & =\sum_{n=1}^{\infty} n\left|a_{n}\right|^{2}=\frac{1}{\pi} \int\left|f^{\prime}\right|^{2} d \omega \\
& =\pi^{-1}\{\text { Area of } f(D), \text { counting the multiplicity of the covering }\} .
\end{aligned}
$$

Proof. The first equality may be computed directly, using the basis $\left\{(n+1)^{1 / 2} z^{n}\right\}_{n=0}^{\infty}$. The others are well known.

COROLlaRY. Let $U$ be a simply connected open set with a smooth Jordan curve for its boundary. Let $g$ be the Riemann map from $U$ to $D$. Then the map $T_{z}$ on $H^{2}\left(\chi_{U}\left|g^{\prime}\right|^{2} \omega\right)$ satisfies $\operatorname{tr}\left[T_{z}^{*}, T_{z}\right]=\pi^{-1} \omega(U)$.

Proof. Taking $g^{-1}=f, T_{z}$ is unitarily equivalent to $T_{f}$ above.

REMARK. If $A_{1}, \ldots, A_{n}$ are each $T_{z}$ on the respective spaces $R^{2}\left(\mu_{i}\right)$, if their spectra are pairwise disjoint and if $\operatorname{tr}\left[A_{i}^{*}, A_{i}\right]=\rho_{i}<\infty$, then the operator $T_{z}$ on $R^{2}\left(\mu_{1}+\cdots+\mu_{n}\right)$ satisfies $\operatorname{tr}\left[T_{z}^{*}, T_{z}\right]=\rho_{1}+\cdots+\rho_{n}$.

It is also necessary to produce trace class intertwining maps. Let $T \in B(H)$. Suppose there is a map $z \rightarrow k_{z}$, from the open set $U$ to $H$, which is conjugate analytic as a map into $H$ in the strong topology, and such that there is a vector $x \in H$ satisfying $\left\langle r(T) x, k_{z}\right\rangle=r(z)$, for all rational functions $r$ with poles off $\operatorname{sp}(T)$, and all $z \in U$. Then the triple $\left(U, k_{z}, x\right)$ will be called an analytic evaluation for $T$, if $T^{*} k_{z}=\bar{z} k_{z}$ for all $z \in U$.

INTERTWINING LEMMA. Let $\left(U, k_{z}, x\right)$ be an analytic evaluation for $T \in B(H)$, and suppose that $x$ is a 1-multicyclic vector for $T$. If $u \in H$, let $\hat{u}(z)=\left\langle u, k_{z}\right\rangle$, for $z \in U$. Let $A \in B(K)$ such that $\operatorname{sp}(A) \subset U$, and let $y \in K$. Define $W: H \rightarrow K, W u=\hat{u}(A) y$. Then $W T=A W$, and $W$ lies in trace class.

Proof. $\hat{u}$ is analytic on an open neighborhood of $\operatorname{sp}(A)$, and so $\hat{u}(A)$ is well defined, say by the Riesz integral. Since $k_{z}$ is an eigenvector for $T^{*}$ with eigenvalue $\bar{z},(T u)^{\wedge}=z \hat{u}$. Thus $W T=A W$. That $W$ lies in trace class results from the fact that the map $z \rightarrow k_{z}$ is strongly conjugate analytic on 
an open neighborhood of $\operatorname{sp}(A)$. Let $\Gamma_{1}$ be a finite set of smooth Jordan curves bounding $\operatorname{sp}(A)$ from $U^{c}$, and let $\Gamma_{2}$ be another such set bounding $\Gamma_{1}$ from $U^{c}$, and $\Gamma_{3}$ a third, bounding $\Gamma_{2}$ from $U^{c}$. Let $\lambda_{i}$ be arc length on $\Gamma_{i}$. Let $H_{i}$ be the closure of the functions $\{\hat{u}: u \in H\}$ in $L^{2}\left(\lambda_{i}\right)$. Let $W_{3}: H \rightarrow H_{3}$ by $W_{3} u=\left.\hat{u}\right|_{\Gamma_{3}} . H_{3}, H_{2}$, and $H_{1}$ admit analytic evaluations. Define $W_{i} u=\left.\hat{u}\right|_{\Gamma_{i}}$ for $u \in H_{i+1}$ for $i=2,1$ and $W_{0} u=\hat{u}(A) y$ for $u \in H_{1}$. $W=W_{0} W_{1} W_{2} W_{3}$, each $W_{i}$ is bounded and it is easy to represent $W_{2}$ and $W_{1}$ as integral operators with square-summable kernels. Thus $W_{2}$ and $W_{1}$ are Hilbert-Schmidt operators, and so $W_{2} W_{1}$ is in trace class [2].

COROllary. Let $\mu$ be a finite measure with compact support. Let $K=H^{2}(\mu)$ and let $E$ be the complement of the unbounded component of the complement of $\operatorname{sp}\left(T_{z}\right)$. $\left[T_{z}^{*}, T_{z}\right]$ is in trace class and $\operatorname{tr}\left[T_{z}^{*}, T_{z}\right] \leqq \pi^{-1} \omega(E)$.

Proof. Let $A=T_{z}$ on $K$. Let $U$ be a simply connected open set with smooth Jordan boundary such that $E \subseteq U$ and $\omega(U)-\omega(E)$ is small. Let $T$ be $T_{z}$ on $H=H^{2}\left(\chi_{U}\left|g^{\prime}\right|^{2} \omega\right)$, where $g$ is as in the corollary to the Computational Lemma. Then $\operatorname{tr}\left[T^{*}, T\right]=\pi^{-1} \omega(U)$. Since $\left|g^{\prime}\right|^{2}$ is bounded away from zero on compact sets in $U$, there exist vectors $k_{z} \in H$ such that $\left(U, k_{z}, 1\right)$ is an analytic evaluation for $T$. Thus the Intertwining Lemma applies. $W 1=1$ is a cyclic vector for $T_{z}$ on $K$, so $W$ has dense range. Thus, the Structure Lemma applies, and so $\operatorname{tr}\left[A^{*}, A\right] \leqq \pi^{-1} \omega(U)$. Thus $\operatorname{tr}\left[A^{*}, A\right] \leqq \pi^{-1} \omega(E)$.

Subspace Dominance Lemma. Let the hyponormal operator $A \in B(H)$ be $n$-multicyclic, with generating vectors $g_{1}, \ldots, g_{n}$. Let $E$ be a compact set containing $\mathrm{sp}(A)$. Let $V$ be the closure of the space spanned by $\left\{r(A) g_{i}\right.$ : $r \in R(E)$, and $1 \leqq i \leqq n\}$. Then $V$ is an invariant space for $A, A V$ is hyponormal, $\operatorname{sp}\left(\left.A\right|_{V}\right) \subseteq E, A V$ is n-multicyclic with generating vectors $g_{1}, \ldots, g_{n}$ and $\operatorname{tr}\left[A^{*}, A\right] \leqq \operatorname{tr}\left[V A^{*}, A V\right]$.

Proof. Unless $\operatorname{tr}\left[V A^{*}, A V\right]<\infty$, there is nothing to prove. Let $\left\{a_{i}\right\}_{i=1}^{\infty}$ be a sequence of points in $E \sim \operatorname{sp}(A)$ which land densely in each component of $\operatorname{sp}(A)^{c}$ which lies entirely in $E$. Let $r_{m}(z)=\prod_{i=1}^{m}\left(z-a_{i}\right)^{-1}$. Let $V_{m}=r_{m}(A) V, V_{0}=V$. Then $V_{m+1} \supset V_{m}$, rank $\left(V_{m+1}-V_{m}\right) \leqq n$, and $V_{m} \nearrow H$ strongly. Thus $\operatorname{tr}\left[V_{m} A^{*}, A V_{m}\right]=\operatorname{tr}\left[V A^{*}, A V\right]$. Let $\left\{e_{k}\right\}_{k}$ be an orthonormal basis for $H$.

$$
\operatorname{tr}\left[V_{m} A^{*}, A V_{m}\right]=\sum_{k}\left[\left\|A V_{m} e_{k}\right\|^{2}-\left\|V_{m} A^{*} e_{k}\right\|^{2}\right]
$$

Thus, since the summands are all nonnegative and approach the corresponding terms for $\operatorname{tr}\left[A^{*}, A\right]$, Fatou's lemma guarantees the desired inequality.

Second Computational Lemma. Let $U_{1}, \ldots, U_{n}$ be open sets with 
disjoint closures, each bounded by finitely many disjoint smooth Jordan curves. Let $U=\bigcup_{i}^{n} U_{i}$ and $H=R^{2}\left(\chi_{U-} \omega\right)$. Then $T_{z}$ on $H$ satisfies $\operatorname{tr}\left[T_{z}^{*}, T_{z}\right] \leqq \pi^{-1} \omega(U)$.

Proof. Let $\left\{G_{i}\right\}_{i=1}^{m}$ be simply connected open sets with smooth Jordan curves as boundaries such that each $G_{i}^{-}$lies in a separate bounded component of $U^{-c}$, and such that $\sum_{i} \omega\left(G_{i}\right)$ is close to the total area of the bounded components of $U^{-c}$. Choose $g_{i}$ so that $T_{z}$ on $H^{2}\left(\left|g_{i}^{\prime}\right|^{2} \chi_{G_{i}} \omega\right)$ satisfies $\operatorname{tr}\left[T_{z}^{*}, T_{z}\right]=\pi^{-1} \omega\left(G_{i}\right)$. Let $T$ be $T_{z}$ on $H, S$ be $T_{z}$ on

$$
R^{2}\left(\chi_{U-} \omega+\sum_{i}\left|g_{i}^{\prime}\right|^{2} \chi_{G_{i}} \omega\right)
$$

$T_{i}$ be $T_{z}$ on $H^{2}\left(\left|g_{i}^{\prime}\right|^{2} X_{G_{i}} \omega\right)$, and let $S^{\prime}$ be $T_{z}$ on $H^{2}\left(\chi_{U^{-}} \omega+\sum_{i}\left|g_{i}^{\prime}\right|^{2} \chi_{G_{i}} \omega\right)$. Let $\tilde{U}$ be the complement of the unbounded component of $U^{i}$. Then

$$
\begin{aligned}
\operatorname{tr}\left[T^{*}, T\right]+\pi^{-1} \sum_{i} \omega\left(G_{i}\right) & =\operatorname{tr}\left[T^{*}, T\right]+\sum_{i=1}^{n} \operatorname{tr}\left[T_{i}^{*}, T_{i}\right] \\
& =\operatorname{tr}\left[S^{*}, S\right] \leqq \operatorname{tr}\left[S^{*}, S^{\prime}\right] \leqq \pi^{-1} \omega(\tilde{U}) .
\end{aligned}
$$

Thus $\operatorname{tr}\left[T^{*}, T\right] \leqq \pi^{-1} \omega(U)$.

It is now possible to prove the Main Theorem.

ThEOREM 1. Let $A \in B(K)$ be hyponormal, with n-multicyclic generating vectors $g_{1}, \ldots, g_{n}$. Then $\operatorname{tr}\left[A^{*}, A\right] \leqq(n / \pi) \omega(\operatorname{sp}(A))$.

Proof. Let $U$ be an open set bounded by a finite number of disjoint smooth Jordan curves, such that $\operatorname{sp}(A) \subset U$, and $\omega(U)-\omega(\operatorname{sp}(A))$ is small. Let $K^{\prime}$ be the space spanned by $\left\{r(A) g_{i}: r \in R\left(U^{-}\right)\right.$, and $\left.1 \leqq i \leqq n\right\}$. Let $A^{\prime}$ be the restriction of $A$ to $K^{\prime} . A^{\prime}$ is hyponormal, and $\operatorname{sp}\left(A^{\prime}\right) \subseteq U$. $\left\{g_{1}, \ldots, g_{n}\right\}$ is a set of $n$-multicyclic vectors for $A^{\prime}$. By the Subspace Dominance Lemma, $\operatorname{tr}\left[A^{*}, A\right] \leqq \operatorname{tr}\left[A^{*}, A^{\prime}\right]$.

Let $T=\oplus \sum_{i=1}^{n} T_{z}$ acting on $H=\oplus \sum_{i=1}^{n} R^{2}\left(\chi_{U} \omega\right)$.

By the Second Computational Lemma, $\operatorname{tr}\left[T^{*}, T\right] \leqq(n / \pi) \omega(U)$. Thus, it only remains to produce an intertwining map between $T$ and $A^{\prime}$ satisfying the conditions of the Structure Lemma.

$R^{2}\left(\chi_{U}-\omega\right)$ has reproducing kernel $k_{z}$ at each $z \in U$. The map $z \rightarrow k_{z}$ is strongly conjugate analytic, and the triple $\left(U, k_{z}, 1\right)$ is an analytic evaluation. Thus by the Intertwining Lemma, the map $W_{i}: R^{2}\left(\chi_{U}-\omega\right) \rightarrow K^{\prime}$ defined by $W f=\hat{f}\left(A^{\prime}\right) g_{i}$ lies in trace class, and $W_{i} T_{z}=A^{\prime} W_{i}$. Let $W: \oplus \sum_{i=1}^{n} R^{2}\left(\chi_{U}-\omega\right) \rightarrow K^{\prime}$ by $W=\sum_{i=1}^{n} W_{i} . W$ lies in trace class, and $W T=A^{\prime} W$. Clearly, the range of $W$ is dense in $K^{\prime}$. Thus

$$
\operatorname{tr}\left[A^{*}, A\right] \leqq \operatorname{tr}\left[A^{\prime *}, A^{\prime}\right] \leqq \operatorname{tr}\left[T^{*}, T\right] \leqq(n / \pi) \omega(U) .
$$


Corollary (Putnam's Theorem [3]). If $A \in B(H)$ is hyponormal, then $\left\|\left[A^{*}, A\right]\right\| \leqq \pi^{-1} \omega(\operatorname{sp}(A))$.

Proof. Let $x \in H,\|x\|=1$, and let $V$ be the closure of the set of vectors $\{r(A) x: r \in R(\operatorname{sp}(A))\} . V$ is an invariant space for $A$. Let $A^{\prime}$ be the restriction of $A$ to $V . A^{\prime}$ is hyponormal.

If $y \in V$ and $a \in \operatorname{sp}(A)^{c},(A-a I)^{-1} y \in V$. Thus $\operatorname{sp}(A) \supseteq \operatorname{sp}\left(A^{\prime}\right)$. It is clear that $A^{\prime}$ is 1 -multicyclic. Thus

$$
\begin{aligned}
\left\langle\left[A^{*}, A\right] x, x\right\rangle & =\|A x\|^{2}-\left\|A^{*} x\right\|^{2} \leqq\|A x\|^{2}-\left\|V A^{*} x\right\|^{2} \\
& =\left\|A^{\prime} x\right\|^{2}-\left\|A^{* *} x\right\|^{2} \\
& =\left\langle\left[A^{*}, A^{\prime}\right] x, x\right\rangle \leqq \operatorname{tr}\left[A^{\prime *}, A^{\prime}\right] \\
& \leqq \pi^{-1} \omega\left(\operatorname{sp}\left(A^{\prime}\right)\right) \leqq \pi^{-1} \omega(\operatorname{sp}(A)) .
\end{aligned}
$$

3. The techniques used above suffice to yield the following results.

THEOREM 2. If the hyponormal operator $A$ has analytic evaluation $\left(U, k_{z}, x\right)$, then $\operatorname{tr}\left[A^{*}, A\right] \geqq \pi^{-1} \omega(U)$.

THEOREM 3. If $A$ is a 1-multicyclic hyponormal operator with generating vector $x$, if $V$ is an invariant space for $A$ containing $x$, and if $A^{\prime}$ is the restriction of $A$ to $V$, then

$$
\operatorname{tr}\left[A^{*}, A\right]+\pi^{-1} \omega\left(\operatorname{sp}\left(A^{\prime}\right) \sim \operatorname{sp}(A)\right) \leqq \operatorname{tr}\left[A^{\prime *}, A^{\prime}\right] .
$$

The corresponding result for $n$-multicyclic hyponormal operators is rather more complicated, and requires a fairly lengthy explanation.

TheOREM 4. For $r \in R(E), T_{r}$ on $R^{2}(E, \mu)$ satisfies

$$
\left[T_{r}^{*}, T_{r}\right] \leqq \frac{1}{\pi} \int_{\operatorname{sp}\left(T_{z}\right)}\left|r^{\prime}\right|^{2} d \omega .
$$

Note that the quantity $\left[T_{r}^{*}, T_{r}\right]$ is a quadratic norm on $R(E)$. The above theorem may be generalized to all functions in the Hilbert space so determined. The following is unknown.

CONJECTURE. There is a measurable function $g$ defined on $\operatorname{sp}\left(T_{z}\right)$ such that $0 \leqq g \leqq 1$, and $\operatorname{tr}\left[T_{r}^{*}, T_{r}\right]=\pi^{-1} \int_{\mathrm{sp}\left(T_{z}\right)}\left|r^{\prime}\right|^{2} g d \omega$ for all $r \in R(E)$.

THEOREM 5. If $R^{2}(E, \mu)$ has analytic evaluation $\left(U, k_{z}, 1\right), F$ is a compact subset of $U, v$ is a finite measure supported on $F$, and $r \in R(E)$, then $\operatorname{tr}\left[T_{r}^{*}, T_{r}\right]$ is the same, whether computed on $R^{2}(E, \mu)$ or on $R^{2}\left(E, \chi_{F c} \mu+v\right)$.

THEOREM 6. If $R^{2}(E, \mu)$ has analytic evaluation $\left(U, k_{z}, 1\right)$, and $0 \leqq g \leqq 1$ is a measurable function such that $g^{-1}([0,1)) \subset U$, then for all $r \in R(E)$, $\operatorname{tr}\left[\mathrm{Tr}^{*}, \mathrm{Tr}\right]$ is not increased when it is computed on $R^{2}(E, g \mu)$ rather than on $R^{2}(E, \mu)$. 
THEOREM 7. Let $A^{2}(U)$ be the Hilbert space of all functions analytic on the open set $U$, and square summable with respect to $\chi_{U} \omega$. Let $f$ be bounded and analytic on $U$. Then $\operatorname{tr}\left[T_{f}^{*}, T_{f}\right]=\pi^{-1} \int_{f(U)} \eta(z, f) d \omega$, where $\eta(z, f)$ is the cardinality of $f^{-1}(z)$.

This theorem may be generalized to the setting of complex manifolds.

For $\mu$ a finite measure with compact support $E$, and $F$ a compact set containing $E$, let $R=R^{2}(F, \mu) \subseteq L^{2}(\mu)$, and for $f \in L^{\infty}(\mu)$, define the "Hankel operator" $H_{f}$ by $H_{f}=(I-R) L_{f} R$. Let $\mathscr{H}=\left\{f \in L^{\infty}(\mu): H_{f}\right.$ is compact\}.

THEOREM 8. If $f \in R(F)$, then $H_{f}$ is a Hilbert-Schmidt operator. $\mathscr{H}$ is a closed subalgebra of $L^{\infty}(\mu)$, and $\mathscr{H}$ contains $L^{\infty}(\mu) \cap R^{2}(F, \mu)+C(E)$.

\section{REFERENCES}

1. T. Kato, Smooth operators and commutators, Studia Math. 31 (1968), 535-546. MR 38 \#2631.

2. C. A. McCarthy, $C_{p}$, Israel J. Math. 5 (1967), 249-271. MR 37 \#735.

3. C. R. Putnam, An inequality for the area of hyponormal spectra, Math. Z. 116 (1970), 323-330. MR 42 \# 5085.

4. - Trace norm inequalities for the measure of hyponormal spectra, Indiana Univ. Math. J. 21 (1971/72), 775-779. MR 45 \#4199.

Department of Mathematics, Belfer Graduate School, Yeshiva University, New YORK, NEW YORK 10033

Department of Mathematics, City University of New York, Bernard M. Baruch College, New York, New York 10010 (Current address of B. I. Shaw) 